\title{
Prader-Willi Syndrome-Improvement of Cognitive Function through Noninvasive Ventilation in Children
}

\author{
Sorina Chindris ${ }^{3 *}$, Ana-Maria Davitoiu ${ }^{1,3}$, Sorina Chindris ${ }^{1,3}$, Iulia Florentina Tincu ${ }^{1,3}$, Vlad \\ Plesca $^{2}$, Doina-Anca Plesca ${ }^{1,3}$, Diana Ionescu ${ }^{1}$, Mihai Dimitriu ${ }^{3}$ and Catalin Gabriel Cirstoveanu ${ }^{3,4}$ \\ ${ }^{1}$ Dr. Victor Gomoiu Children Clinical Hospital, Bucharest, Romania
}

${ }^{2}$ Colentina Clinical Hospital, ENT Department, Bucharest, Romania

${ }^{3}$ Carol Davila University of Medicine and Pharmacy, Bucharest, Romania

${ }^{4}$ Marie Curie Emergency Clinical Hospital, Anesthesia and Intensive Neonatal Care Unit, Bucharest, Romania

*Corresponding author: Sorina Chindris - "Carol Davila” University of Medicine and Pharmacy, "Dr. Victor Gomoiu" Children

Clinical Hospital, Bucharest, Romania

\section{ARTICLE INFO}

Received: 蔧 December 12, 2020

Published: 幽 December 22, 2020

Citation: Sorina C, Ana-Maria D, Sorina C, Iulia Florentina T, Vlad P, et al., PraderWilli Syndrome-Improvement of Cognitive Function through Noninvasive Ventilation in Children. Biomed J Sci \& Tech Res 32(5)2020. BJSTR. MS.ID.005300.

Keywords: Children, Prader-Willi Syndrome; Attention and Concentration Disorders; Sleep Disturbances; Sleep Apnea; Polysomnography; Noninvasive Ventilation; Romania

Abbreviations: PWS: Prader-Willi Syndrome; CPAP: Continous Positive Airway Pressure; QI/QD: Intelligence Quotient; CDC: Center of Disease Control; PSG: Polysomnography

\section{ABSTRACT}

Background: PWS is a rare genetic disease characterized by a variety of physical, psychological and physiological changes. The prevalence of PWS is estimated to be between 1:12000 - 1:25000 live births, no matter the gender or race. The pathology occurs in a percentage $<1 \%$ of congenital diseases associated with mental retardation. The disease is diagnosed during the neonatal period and in infants with the help of the known association of facial dysmorphism with marked muscle hypotonia and eating disorders. In evolution, patients with PWS have sleep-related respiratory disorders with severity changes over time, depending on the clinical stage of the disease.

Case report: Patient diagnosed in the neonatal period with PWS (intrauterine growth retardation, craniofacial dysmorphism, hypotonia, eating difficulties) and confirmed by molecular genetics tests; evaluated in our clinic at the age of 4 years. The polysomnography showed severe obstructive sleep apnea syndrome associated with severe intermittent hypoxemia. The patient suffers from mental retard with $Q D=45$, attention and concentration disorders and aggression traits. It is decided to initiate nocturnal noninvasive ventilation, CPAP therapy throughout sleep period. The growth hormone substitution therapy is continued, and the patient is included in a cognitivebehavioral therapy program.

Conclusions: The evaluation done at 12 months after the initiation of the noninvasive ventilation at home highlights the increase in both the concentration ability and the memory capacity, the improvement of the relationship abilities, the increase of the school performances, the reduction of the impulsivity traits associated with the improvement of QI/QD. The authors try to establish a correlation between reducing the hypoxemia through noninvasive nocturnal ventilation and the improvement in the cognitivebehavioral function.

\section{Introduction}

Prader-Willi Syndrome is a complex congenital disorder characterized by neonatal hypotonia, short stature, childhood onset obesity, craniofacial dysmorphism, hypogonadism, developmental delay and behavioral problems [1-5]. Patients with Prader-Willi syndrome have a high number of risk factors for developing respiratory problems during sleep. They may develop sleep fragmentation, breathing difficulties, snoring or sleep apnea [1-5]. Sleep related respiratory problems appear secondary to characteristic features of the disease such as muscular hypotonia, obesity, craniofacial dysmorphism, airway sticky secretions, etc. [1-4]. Most patients with Prader-Willi Syndrome have one of the four identified genetic errors. The most common genetic change encountered in these patients (approximately 70\%) is paternal 
deletion of chromosome 15. Maternal uniparental disomy is seen in $25 \%$ of the patients, $2-5 \%$ have imprinting errors and below $1 \%$ translocation [6-9].

Recent data suggest that there is an increase in the number of patients diagnosed with Prader-Willi Syndrome due to maternal disomy possibly secondary to the increased in maternal age at the time of conception [6-10]. Genotypic changes result in polymorphic phenotypic changes that associate various degrees of physical, physiological and psychological damage [10]. Physical factors include the association of infantile hypotonia with onset during the intrauterine life (reduced fetal movements), hypogonadism, craniofacial dysmorphism, scoliosis, obesity and short stature [1113]. Patients have characteristic physiological traits (muscular hypotonia, metabolic imbalance, sleep related respiratory problems, excessive daytime sleepiness), associated with psychological traits with various degrees of impairment (tantrums, hyperphagia, obsessive-compulsive behavior, irritability, low frustration threshold, intellectual impairment) [11-15]. Phenotypic changes are the consequence of genetic damage in PWS.

Moreover, these patients frequently associate sleep related respiratory syndrome [11]. The authors aim to draw attention to the evolution of a patient diagnosed with Prader-Willi Syndrome who shows a dramatic improvement of both the cognitive function and the psychological component as a result of the correction of nocturnal hypoxemia.

\section{Case Report}

A male patient diagnosed with PWS during the neonatal period was referred to the cardiology department of our hospital, Bucharest, Romania, in September 2019, at the age of 4 years ols, for the investigation of an atrial septal defect. We know that he is a second-degree child of young parents (mother: 24 years, father: 26 years), healthy, not consanguineous, without congenital abnomalies, metabolic diseases or reproductive disorders. Pregnancy is monitored (the mother reports reduced fetal movements compared to the first pregnancy), it has intrauterine growth retardation in the third trimester of pregnancy. He is extracted by cesarean section at 39 weeks of gestation, birth weight: 2400 grams, length: $49 \mathrm{~cm}$, Apgar score 4 - shows marked muscular hypotonia at birth. In dynamics, the impossibility of natural feeding or bottle feeding is ascertained and feeding by gavage for 2 weeks is necessary. The association of marked muscular hypotonia with elements of craniofacial dysmorphism (dolichocephaly, lower implanted ears, micrognathia, pointed palatal arch, elongated facies, high forehead, narrow eyelid slits which are almond shaped, short nose) raised the suspicion of PWS.

Chromosomal analysis is performed that shows normal karyotype, $46 \mathrm{XY}$. The investigations are completed with molecular cytogenetic analysis FISH. The FISH technique is performed on metaphase chromosomes and uses Leica probes specific to the $15^{\text {th }}$ chromosome, SNRPN (SNRPN probe specific to the Prader-Willi locus 15q11) and PML (localized control probe 15q24). Thus, the result of the analysis reveals $46 \mathrm{XY}$, ish(15) (q1q13)(D15S10)x2. In this context, molecular analysis is performed by genomic DNA study, MS-MLPA test, to verify methylation at the Prader-Willi/ Angelman locus. The analysis bulletin confirms the existence of a methylation defect in the critical area 15q11-q13 and supports the diagnosis of PWS. In conclusion, the association of the features of craniofacial dysmorphism with generalized hypotonia and characteristic genetic changes allow the establishment of the diagnosis of PWS in the neonatal period. During the first year of life, the child registers retardation of stature-weight growth: at 1 year old he has weight of 8200 grame (percentile -1.75) and height of $73 \mathrm{~cm}$ (percentile -1.25) and delay in neuromotor development (holds his head at 6 months, sits at 9 months, walks without support at 1 year and 6 months).

In the context of the characteristic genetic damage and the clinical evolution, at the age of 1 year and 4 months, the multidisciplinary team led by the endocrinologist includes the patient in the National Health Programme for Rare Diseases, in order to administer the substitution treatment with somatotropic growth hormone. The patient has been receiving hormone replacement therapy since the age of 1 year and 4 months including now (2016-2020). At the first hospitalization in our hospital, the clinical examination of the 4 years old patient reveals:

a) Anthropometric indices: Height $=107 \mathrm{~cm}$ (percentile +0.75 ), Weight $=24 \mathrm{~kg}$ (over the $3^{\text {rd }}$ percentile), $\mathrm{BMI}=21.4 \mathrm{~kg} /$ $\mathrm{m}^{2}$ - 99th percentile for age and sex according to CDC - after administration of growth hormone for 3 years.

b) Craniofacial dysmorphism: round skull, high forehead, elongated facies, bitemporal retraction, narrow eyelid slits which are almond-shaped, small and short nose, small mouth, thin upper lip, lowered mouth corners, micrognathia, oral respiration.

c) Dental dystrophies, thick salivary secretions, hypertrophy of the I/ IV tonsils.

d) Small and short hands, fingers with distal conical phalanx;

e) Hyperphagia;

f) Hypoplazic scrotum, operated bilateral cryptorchidism;

g) Artherial blood pressure: $100 / 63 \mathrm{mmHg}$, heart rate: 100 bpm.

h) Moderate psychomotor retardation QI/ QD $=45-50$.

i) Hyperkinetic, attention and behavior disorders.

\section{Discussion}

The patient shows snoring and sleep apnea, sleep fragmentation, numerous awakenings durind sleep, daytime sleepiness, he is difficult to wake up in the morning. The mother observes the 
exacerbation of the hyperkinetic syndrome, the decrease in school performances, the low concentration capacity, the deterioration of the acquisitions (cognitive sphere), the increase of aggressiveness and the impulsivity within the family and the entourage.

The patient is evaluated multidisciplinary:

1) ENT consultation - deviation of the nasal septum, chronic hypertrophic rhinitis;

2) Cardiologic consultation - echocardiography and electrocardiogram within normal limits;

3) Abdominal ultrasound within normal limits.

4) Psychological consultation $(\mathrm{QI} / \mathrm{QD}=45-50)$

5) Neurological consultation - Delay in neuromotor and language development. Moderate mental retardation. Attention disorders. Hyperkinetic syndrome. Sleep apnea in observation.

The pediatric sleep questionnaire is applied in front of a 4 year old patient with PWS who has respiratory sleep disorders and behavioral disorders (hyperactivity, impulsivity, attention disorder). The patient scores 0.81 which indicates an increased risk for obstructive sleep apnea. It is decided to perform full-night attended polysomnography and this finds a severe form of obstructive sleep apnea syndrome. Cardiorespiratory polysomnography is performed with the Alice 6LDx polysomnographic system, with registration F3M2, F4M1, C3M2, C4M1, 01M2, 02M1, electrooculogram, chin EMG, nasal and oral flow, snoring microphone, SaO2, heart rate, respiratory rate, thoracic and abdominal belths, electrocardiogram. AASM VIII4.B criteria (3\% desaturation) were used to score the events. a. Sleep parameters: Recording duration: 622.5 minutes, with a sleep efficiency of $80.9 \%$. NREM stage 1 was $16.5 \%$, NREM 2 was $46.3 \%$, NREM 3 was $24.2 \%$ and REM stage was $12.9 \%$. There were 29 micro-awakenings with an index of 3.5 / h.

b. Respiratory parameters: During the analyzed route, respiratory events such as obstructive apnea and obstructive hypopnea and snoring were recorded.

$\mathrm{RDI}=40.6 / \mathrm{h}$, desaturation index: 42.9/ h.

Average heart rate during sleep: $117 \mathrm{bpm}$.

Minimum SaO2 during sleep: 72\%.

Average SaO2 during NREM sleep: 96\% and in REM sleep : 94\%.

Time spent with $\mathrm{SaO} 2<90 \%: 8$ minutes

Figure 1 shows polysomnographic findings during attended full-night PSG - disrupted sleep, poor sleep quality, obstructive apnea and hypopnea events during entire night. Figure 2 shows Severe Obstructive Sleep Apnea Syndrome with low desaturations following apnea episodes in our PWS patient during PSG. Figure 3 shows overnight hipnogram during PSG investigation-with a severe form of sleep related respiratory findings, poor sleep quality and modified percent of sleep stages. The result of the polysomnography requires the initiation of noninvasive ventilation with continuous positive airway pressure during sleep. CPAP titration is practiced under polysomnographic monitoring to establish the optimal parameters of noninvasive ventilation. We initiate CPAP therapy at $10 \mathrm{~cm} \mathrm{H}_{2} \mathrm{O}$ pressure, throughout sleep, 20 minutes ramp, C-flex-3, Pico S nasal mask, with humidifier as needed.

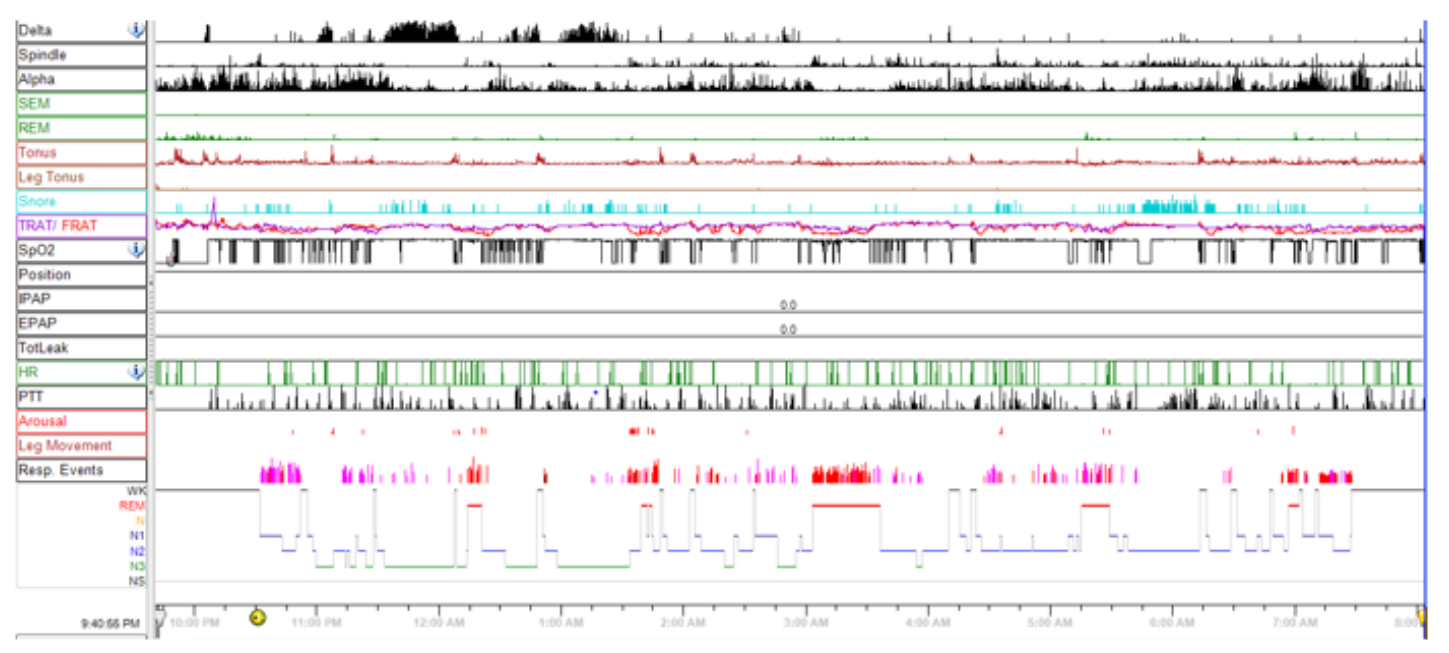

Figure 1: Polysomnographic findings during attended full-night PSG. 


\section{Summary

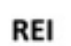 \\ 40.6 \\ OAI \\ 23.7 \\ CAI \\ 0.0 \\ Lowest Desat \\ 72}

REI is the number of respiratory events per hour. OAl is the number of obstructive apneas per hour. CAl is the number of central apneas per hour. Lowest Desat is the lowest blood oxygen level that lasted at least 2 seconds.

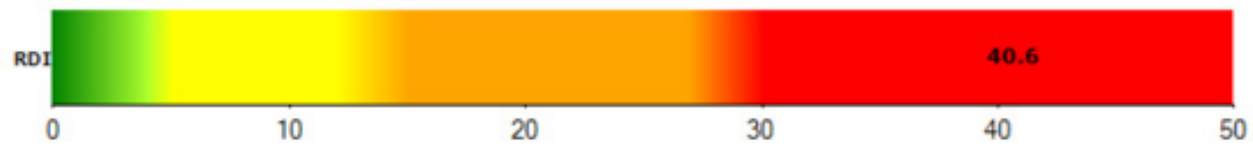

Figure 2: Severe Obstructive Sleep Apnea Syndrome with low desaturations following apnea episodes in our PWS patient during polysomnography.

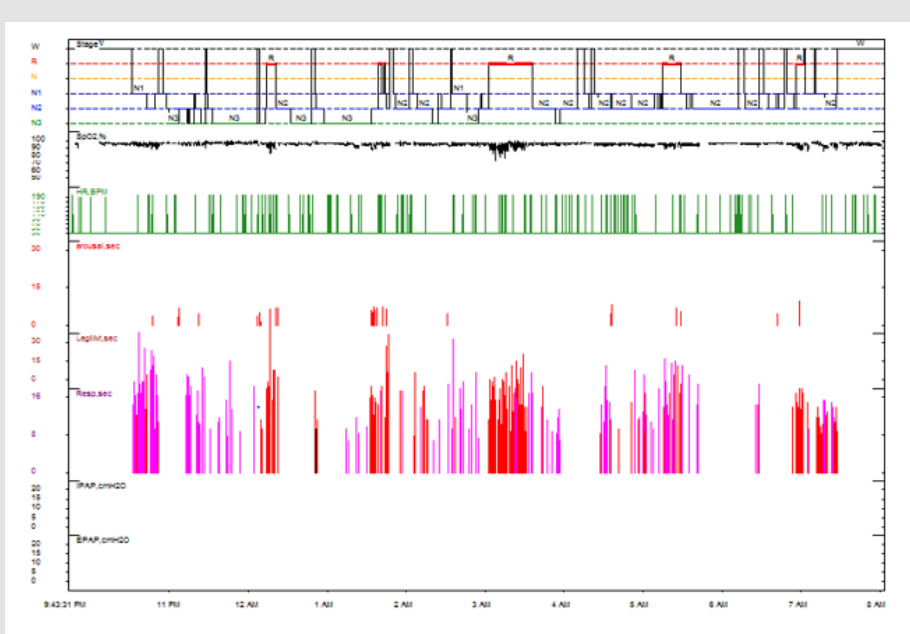

Figure 3: Overnight hipnogram during PSG investigation.

Due to manual titration, the pressure established managed to treat episodes of sleep apnea and hipopnea and to improve the quality of sleep. Patient compliance is good for using CPAP at home. He uses the CPAP every night with an appropriate number of hours / night (average 7-8 hours/ night) and good tolerance. Figure 4 shows patient compliance and hours of CPAP use at home between $29^{\text {th }}$ february 2020 - 11th september 2020, data obtined from reading compliance CPAP card According to the monitoring protocol of the patient with noninvasive ventilation, the child is evaluated periodically, at intervals of one month, 3 months, 6 months. As a result of reading the compliance card, it is decided to maintain the noninvasive ventilation parameters at the same values. The child is periodically monitored multidisciplinary: endocrinology, psychology, speech therapy and benefits from cognitive-behavioral therapy. He receives nutrition according to the recommendations of a nutritionist specialized in the nutrition of the patient with PWS.

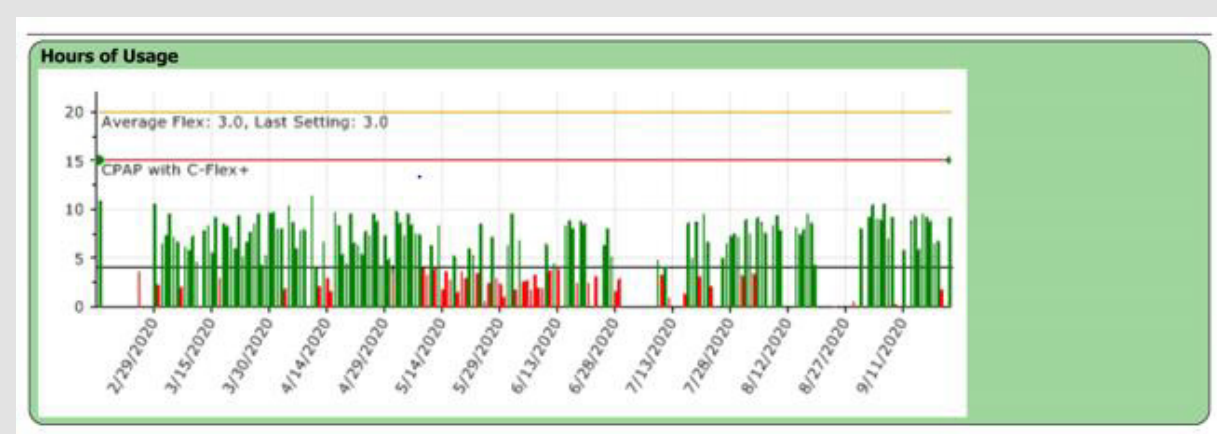

Figure 4: Patient compliance and hours of CPAP use at home between $29^{\text {th }}$ february 2020-11th september 2020, data obtained from reading compliance CPAP card. 
Growth hormone substitution is administered in doses adjusted according to biological parameters. After 12 months of CPAP therapy, the patient arrives at the hospital for clinical-paraclinical assessment. The clinical examination at submision shows a patient aged 5 years, height $=115 \mathrm{~cm}$ (percentile +1.75$)$, weight $=24.5 \mathrm{~kg}$ (percentile +2.75 ); $\mathrm{BMI}=18.5 \mathrm{~kg} / \mathrm{m}^{2}-97^{\text {th }}$ percentile for age and sex according to CDC. They mention rarely snoring and rare episodes of apnea. The mother acknowledges the improvement in the quality of sleep and the quality of life by the improvement of the relationship abilities and of the school performances. The psychological examination with QD 60 determination is repeated and it highlights the increase of concentration and memory capacity, the improvement of attention, the improvement of the relationship, the improved interpersonal contact, the increase of the academic performances, the improvement of the impulsivity traits. It is decided to continue non-invasive ventilation therapy. When re-titrating under polysomnographic monitoring, the CPAP pressure is adjusted to $11 \mathrm{~cm} \mathrm{H}_{2} \mathrm{O}$, during the whole sleep, minimum 7-8 hours/ night.

\section{Conclusion}

The authors note the improvement in cognitive performance supported by increased QD in a patient diagnosed with PWS. The idea that reducing hypoxia and improving sleep quality (confirmed by polysomnographic findings and supported by anamnestic and psychological examination) increase the quality of life and academic performance in the PWS patient noninvasively ventilated at home, is credited. The monitoring of a patient with PWS will be performed in a multidisciplinary center, with the permanent intervention of the endocrinologist in order to adjust the dose of growth hormone, with regular polysomnography assessments. The patient will be assisted by a specialized nutritionist and will benefit from the competence of behavioral therapists. The authors work in a highly addressable clinic for patients with Prader-Willi Syndrome and benefit from a high-performance laboratory and specialized staff in the sleep study. The authors aim to expand the group of patients with PWS who require nocturnal noninvasive ventilation to confirm a correlation between hypoxia secondary to respiratory sleep disorders and improved academic and behavioral performance after 12 months post initiation of noninvasive ventilation with continuous positive airway pressure.

\section{References}

1. Hsing Yu L, Shuan Pei L (2007) Polysomnographic characteristics in patients with Prader-Willi Syndrome.Pediatric Pulmonology 42(10): 888-887.

2. Gutierrez F, Mendez M (2020) Prader-Willi Syndrome. Stat Pearls.

3. Deal CL, Rogol AD (2020) Growth hormone treatments and cognitive functioning in children with Prader-Willi syndrome.European Society of Endocrinology 182(6): 21-25.

4. Wattendorf DJ, Muenke M (2005) Prader-Willi syndrome. Am Physician 72(5): 827-830.

5. Nixon GM, Brouillette RT (2002) Sleep and breathing in Prader-Willi syndrome. Pediatr Pulmonol 34(3): 209-217.

6. Camfferman D, Doug McEvoy R, O’Donoghue F, Lushington F (2008) Prader-Willi Syndrome and excessive daytime sleepiness. Sleep Medicine Reviews 12(1): 65-75.

7. State MW, Dykens EM (2000) Genetics of childhood disorders: XV. Prader-Willi syndrome: genes, brain, and behavior. Journal of the American Academy of Child and Adolescent Psychiatry 39(6): 797-800.

8. Everman DB, Cassidy SB (2000) Genetics of childhood disorders: XII. Genomic imprinting: breaking the rules. Journal of the American Academy of Child and Adolescent Psychiatry 39(3): 386-389.

9. Smith A, Hung D (2017) The dilemma of diagnostic testing for PraderWilli syndrome. Translational Pediatrics 6(1): 46-56

10. Whittington JE, Holland AJ, Butler JV (2007) Changing rates of genetic subtypes of Prader-Willi syndrome in the UK. Eur J Hum Genet 15(9): 127-130.

11. Camfferman D, Lushington K, O’Donoghue F (2020) Obstructive Sleep Apnea Syndrome in Prader-Willi Syndrome: An Unrecognized and Untreated Cause of Cognitive and Behavioral Deficits. Neuropsychol 16(3): 123-129.

12. Sedky K, Bennett D, Pumariega A (2014) Prader Willi Syndrome and Obstructive Sleep Apnea: Co-occurrence in the Pediatric Population. Journal of Clinical Sleep Medicine 10(4): 403-409

13. Gillett ES, Perez IA (2016) Disorders of Sleep and Ventilatory Control in Prader-Willi Syndrome. Diseases 4(3): 23.

14. Polytarchou A, Katsouli G, Tsaoussoglou M, Panaghiotopoulou-Gartagani P, Kaditis AG, et al. (2018) Obstructive events in children with PraderWilli syndrome occur predominantly during REM sleep. European Respiratory Journal 52(62): 552-559.

15. Reddy LA, Pfeiffer SI (2007) Behavioral and emotional symptoms of children and adolescents with Prader-Willi Syndrome. J Autism Dev Disord 37(5): 830-839.
ISSN: 2574-1241

DOI: 10.26717 /BJSTR.2020.32.005300

Sorina Chindris. Biomed J Sci \& Tech Res

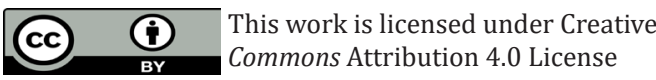

Submission Link: https://biomedres.us/submit-manuscript.php

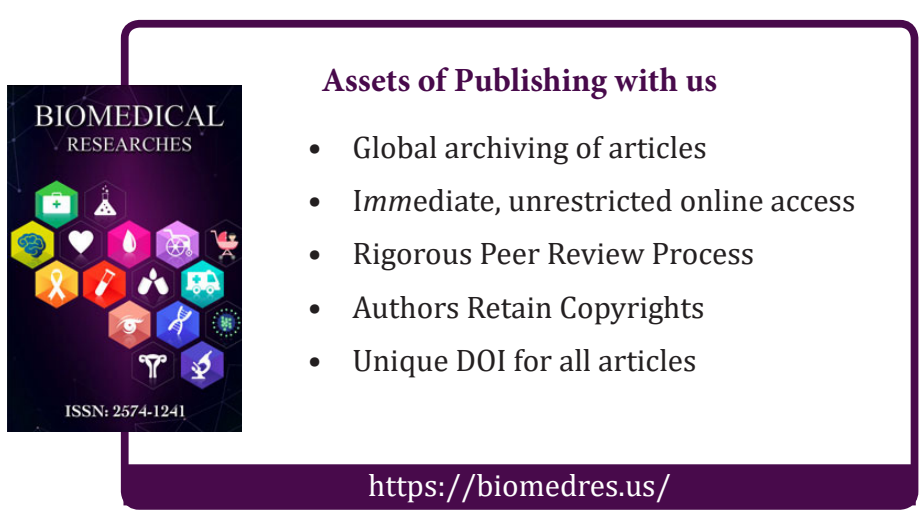

